\title{
LA BÚSQUEDA DEL CENTRO DE ORIGEN EN BIOGEOGRAFÍA HISTÓRICA
}

\author{
N. López Martínez*
}

\begin{abstract}
RESUMEN
En este trabajo se estudia la aplicación del concepto de centro de origen de un taxón en Biogeografía Histórica a través de dos predicciones de un modelo biogeográfico frecuentemente usado, que denominamos "modelo simétrico": $1^{\circ}$ ) aparición local de las nuevas especies, seguida de $2^{\circ}$ ) expansión gradual de su área inicial de distribución. La primera predicción sólo se cumple en especies insulares: a) la aparición local de nuevas especies no ha sido observada hasta ahora en regiones no insulares; b) el centro de origen de especies no insulares no ha podido ser localizado con precisión, y c) los criterios para inferirlo no convergen. La segunda predicción se cumple en especies invasoras, que tras franquear barreras preexistentes expanden el área linealmente, a velocidad aproximadamente constante, mostrando para ello una capacidad intrínseca no ligada a factores externos. El modelo simétrico asume una dinámica biogeográfica simétrica en el tiempo: $1^{\circ}$ ) origen local, $2^{\circ}$ ) expansión gradual, $3^{\circ}$ ) estasis, $4^{\circ}$ ) contracción gradual, y $5^{\circ}$ ) extinción. Diversos datos refutan el modelo simétrico: a) las especies endémicas, únicas que muestran una distribución localizada, no muestran capacidad de expansión; b) el "ciclo del taxón" en especies insulares muestra que el área de la especie colonizadora tiende siempre a reducirse según va diferenciándose; c) las entradas y salidas de especies-guías del registro fósil son asimétricas, con pautas de aparición muy rápida a escala regional (cuasi-sincrónica), y de extinción muy lenta y diacrónica a lo largo de su área de distribución; y d) la historia paleobiogeográfica de muchos taxones es asimétrica, con una amplia área de distribución inicial y una tendencia lenta a su contracción y fragmentación antes de la extinción. Por tanto, los datos refutan la aplicabilidad del modelo simétrico y del concepto de centro de origen, y apuntan a un modelo de dinámica biogeográfica asimétrico en el tiempo: $1^{\circ}$ ) aparición súbita del taxón en una amplia área, $2^{\circ}$ ) estasis, $3^{\circ}$ ) lenta contracción, y $4^{\circ}$ ) extinción, frecuentemente precedida de fragmentación del área.
\end{abstract}

Palabras clave: centro de origen, biogeografía histórica, biogeografía insular, paleobiogeografía.

\section{ABSTRACT \\ The search for the centre of origin in Historical Biogeography}

We study the application of the concept 'centre of origin' in Historical Biogeography through the predictions of a commonly used biogeographic model, here defined as "symmetric model": i) local appearance of new species, followed by ii) expansion of the range of the distribution area of the new species. The first prediction occurs only in island species: a) the local appearance of new species has not been documented in not insular regions; $b$ ) the centre of origin cannot be accurately located for not insular species, and c) the criteria for infer it do not converge. The second prediction applies only for invasive species, after opening pre-existent barriers, showing their intrinsic capacity for linear expansion of the area at approximately constant speed and not controlled by external factors. The symmetric model assumes a symmetrical biogeographic dynamics: $1^{\text {st }}$ ) local origin, $2^{\text {nd }}$ ) gradual expansion, $3^{\text {rd }}$ ) stasis, $4^{\text {th }}$ ) gradual contraction and $5^{\text {th }}$ ) extinction. Diverse data refute this symmetrical model:

* Dept. Paleontología, Fac. C. Geológicas. Universidad Complutense de Madrid. 28040 MADRID (España). lopezmar@geo.ucm.es 
a) the endemic species, the only ones who have a local distribution, show incapacity for expansion of their area;

b) the "taxon cycle" in insular species shows that the area of the colonizing species always tend to decrease while differentiation occurs;

c) the entries and exits of the guide species in the fossil record show an asymmetric pattern, with very rapid, quasi-synchronic appearance at regional scale and very slow, diachronic extinction along their distribution area; and

d) the chronological asymmetry of the paleobiogeographic history of taxa, showing a wide distribution area at the beginning and a slow trend towards area contraction and fragmentation at the end, before extinction.

Therefore, the data refute the applicability of the centre of origin concept and point to an asymmetric model of biogeographic dynamics: $1^{\text {st }}$ ) sudden appearance of the taxon in a wide area, $\left.2^{\text {nd }}\right)$ stasis, $3^{\text {rd }}$ ) slow contraction and $4^{\text {th }}$ ) extinction, frequently after previous fragmentation of the area.

Key words: centre of origin, historical biogeography, insular biogeography, paleobiogeography.

\section{Introducción}

En los años ochenta la Biogeografía se afianzó como disciplina científica mientras el foco de los estudios biogeográficos pasó de los taxones a las áreas. El método para plantear y poner a prueba las hipótesis biogeográficas tiene ahora en cuenta no sólo la movilidad de los organismos (dispersión) sino ante todo la de las áreas (vicarianza). Las relaciones entre bioprovincias, basadas en datos corológicos de áreas de distribución de los taxones, son ahora hipótesis verificables que buscan explicaciones en factores ambientales (Biogeografía Ecológica) o históricos (Biogeografía Histórica). Aunque para muchos autores las disciplinas históricas son las menos avanzadas dentro de las ciencias, la Biogeografía Histórica constituye una inevitable encrucijada donde se confrontan modelos geodinámicos e hipótesis filogenéticas para explicar las observaciones corológicas y ecológicas.

Mucho antes de que los planteamientos evolucionistas de Wallace y Darwin fueran aceptados, en Biogeografía Histórica era clásico el concepto de centro de origen. Desde Linneo y Cuvier, este concepto ya suponía que todas las especies ocuparon inicialmente un área restringida (su lugar o centro de origen) y se extendieron posteriormente utilizando diversos medios de dispersión hasta expandirse por un área más extensa. Sin duda, la generalización de la Teoría Evolutiva contribuyó a la aceptación y aplicación de este concepto. Sin embargo, su uso en Biogeografía supuso siempre un problema, hasta que escuelas de Biogeografía Histórica como la Panbiogeografía y la Biogeografía cladista han propuesto el abandono de este concepto, rechazado explícitamente como inverificable por Croizat et al. (1974). Con un enfoque sistémico, el estudio de las regiones con sus biotas no precisaría de tal concep- to individual, orientándose en su lugar hacia el descubrimiento de congruencias de las pautas observadas (Platnick \& Nelson, 1978; Nelson \& Rosen, 1981; Nelson \& Platnick, 1981; Morrone \& Crisci, 1995; Crisci et al., 2000; Humphries, 2002). Análogamente, el concepto de antepasado dentro de las hipótesis filogenéticas encuentra similares dificultades, por lo que también ha sido tachado de inverificable y no científico.

Sin embargo, sigue habiendo escuelas de Biogeografía Histórica que consideran el concepto de centro de origen pertinente para la reconstrucción de escenarios y procesos biogeográficos particulares, como las de Biogeografía filogenética, Filogeografía, análisis de parsimonia de Brooks, de dispersión-vicarianza de Ronquist e incluso en Biogeografía cladista (análisis del área ancestral de Bremer), donde se ha propuesto el concepto de "área ancestral" en lugar del de centro de origen, dadas las dificultades metodológicas y operativas encontradas al tratar de precisar su localización (al igual que el concepto de "grupo externo" en filogenia permite señalar un grupo ancestral sin tener que precisar un antepasado concreto). Sin embargo, estos conceptos ampliados se basan en principios históricos similares a los conceptos restringidos y requieren igualmente de criterios indirectos para su inferencia, a falta de observaciones directas sobre los procesos de origen de las especies.

Precisamente en la Península Ibérica se ha situado el centro de origen o el área ancestral de numerosas especies (ver p. ej. Oosterbroek y Arntzen, 1992), por lo que nuestra región está favorablemente situada para explorar las posibilidades de aplicación de este concepto, lo que abordamos en este trabajo. Emplearemos para ello el método hipotéticodeductivo que supone: 1) plantear un modelo concreto en el que opere el concepto de centro de ori- 
gen, y 2) confrontar algunas de sus deducciones con datos empíricos sobre procesos de modificación del área de distribución de especies recientes o extinguidas. Utilizaremos para ello 1) datos procedentes de la observación directa de la dinámica biogeográfica de especies actuales, y 2) datos de procesos inferidos a partir de pautas observadas sobre organismos vivos y en documentos paleontológicos.

\section{El concepto de centro de origen de una especie}

El concepto de centro de origen es un postulado que se asocia a menudo a la escuela evolucionista de Darwin y Wallace. Sin embargo es un concepto mucho más antiguo, admitido tanto por autores evolucionistas como no evolucionistas desde Linneo (Morrone \& Crisi, 1995). La formulación de este postulado por Cuvier (1798) sigue estando hoy vigente:

"Parece que en el principio cada especie de animal, e incluso de planta, no existía más que en una región determinada, de donde se ha distribuido según los medios que su configuración le daba. Todavía hoy varias de ellas parecen haber estado limitadas alrededor de semejantes centros de origen, o por los mares cuando no podían nadar ni volar, o por temperaturas que no han podido soportar, o por montañas que no han podido franquear..." (trad. López-Martínez y Truyols, 1994, pg. 288).

La expresión bíblica del Génesis “creced y multiplicaos..." expresa la idea de que las especies se expanden por un espacio previamente no poblado. Análogamente, la historia de los procesos antrópicos muestra numerosos ejemplos de invasiones y colonizaciones imperiales. Croizat (1960) encuentra la idea explícita en la bibliografía desde finales del siglo XV, cuando los clérigos españoles se preguntaban por el lugar de origen de los indios americanos a los que consideraban procedentes de otro continente (idea que los modernos antropólogos confirman). Posteriormente, el concepto de "centro de origen" se ha visto reforzado en el marco evolucionista, como muestran las formulaciones de Darwin (1859, pg. 352):

“... la simplicidad de la idea de que cada especie fue primero producida en una única región cautiva la mente. Quien la rechaza, rechaza la vera causa de generación ordinaria con subsiguiente migración, y pide la actuación de un milagro...";

Darwin asimila aquí el concepto de movilidad dentro del área de distribución de la especie (disemina- ción de diásporas, migración), al de expansión del área a zonas no ocupadas previamente (dispersión fuera del área de distribución de la especie); y Udvardy (1969, pg. 7):

"Cada especie animal se originó de unos pocos antecesores en un área limitada; si una especie particular está actualmente muy extendida, debe necesariamente haber alcanzado partes de su presente área en un periodo anterior" (en Croizat et al., 1974).

El concepto de centro de origen ha sido ilustrado con muchos ejemplos de diásporas (gérmenes resistentes) capaces de diseminarse a grandes distancias, pero son conceptos distintos. El centro de origen va ligado al concepto de especiación o formación de una nueva especie a partir de una pequeña población diferenciada que ha quedado reproductivamente aislada, ya sea en una pequeña área marginal (modelo alopátrico) o dentro del área de la especie ancestral (modelo simpátrico). Tras la divergencia, la nueva especie invadiría un área más extensa y puede reemplazar a la especie ancestral y/o coexistir con la especie hermana.

Sin embargo, los detractores del uso del concepto de centro de origen cuestionan su aplicabilidad o incluso la realidad de su existencia, dado que en muchos casos el área concreta de procedencia de las especies no ha podido ser precisada. Por ejemplo, Olson (1971, pg. 738, en Croizat et al., 1974):

"Los conceptos de centro de origen y dispersión están profundamente arraigados en el pensamiento biogeográfico y sostenidos por tantas evidencias en los casos mejor conocidos, que otros conceptos han recibido poca atención. Pero en muchos casos especificos surge la punzante cuestión de cuál fue el centro de origen o de si hubo un centro. Lo que se ve comúnmente en el registro fósil parece sugerir que la evolución siempre ocurrió en algún otro lugar"

También Croizat et al. (1974, pg. 273), tras una revisión de numerosos intentos de aplicación, rechazan el concepto de centro de origen:

"El conflicto de opiniones resultante de diferentes apriorismos plantea la cuestión de la aplicabilidad del concepto e incluso de la existencia de un centro de origen como se concibe en esas discordantes contribuciones".

Muchos autores siguen esforzándose con diversas metodologías y casos bien documentados en localizar el lugar de origen de los taxones, llámese área ancestral, área fuente o punto de partida (Bremer, 1992; Enghoff, 1995; Hausdorf, 1998). Sin 
embargo, su localización sigue encontrando numerosas dificultades, hasta el punto que Carlton (1996) propone el uso del término "especie criptogénica" (de origen desconocido) para el elevado número de casos en que no es posible determinar si una especie actual es nativa o invasora de una región. Veremos las dificultades que se encuentran los autores al establecer y aplicar criterios para inferir el centro de origen de las especies que estudian.

\section{Identificación del centro de origen de un grupo}

Diversos criterios se aplican en la práctica a la localización del centro de origen:

CRITERIO 1): el área de máxima diversidad. El centro de origen de un grupo se localizaría en la zona en la que presente mayor número de variantes (taxones, morfos, alelos, etc.). Este criterio antiguamente propuesto por Willis se usa actualmente en Filogeografía, donde la teoría del centro génico localiza el centro de origen en el área de mayor diversidad génica de un taxón (Avise, 2000). Se basa en la suposición de que en dicha área fuente el taxón habría permanecido más tiempo y por tanto sufrido allí más eventos de modificación. Este criterio implica en primer lugar, que el taxón ocupa permanentemente su área de origen; en segundo lugar, que los procesos de modificación serían esencialmente simpátricos; y en tercer lugar, que la mayoría de las poblaciones sucesivamente modificadas permanecerían en el área ancestral cada vez más rica en variantes, en vez de dispersarse. Aunque ninguna de estas implicaciones ha sido observada, el criterio sigue siendo ampliamente utilizado aún cuando produzca evidencias contradictorias (por ejemplo, la existencia de más de un centro; o la discrepancia entre el centro de mayor riqueza de especies y el de mayor polimorfismo; ver Sáiz, 1973, pg. 290; Hengeveld, 1989, pg. 95). Alternativamente, se ha observado que un mayor polimorfismo está ligado a una mayor heterogeneidad ambiental, no al área original (Van Valen, 1965; McDonald \& Ayala, 1974; Doadrio et al., 1996).

CRITERIO 2): el área de mayor antigüedad paleontológica del grupo. El centro de origen de un grupo se localizaría en la zona en la que se encuentren sus fósiles más antiguos. Los sesgos de conservación de los restos de organismos y de accesibilidad de los fósiles para su estudio afectan al uso de este criterio. Ilustra el caso los recientes hallazgos de homínidos mio-pliocenos centroafricanos a varios miles de kilómetros de su hallazgo más antiguo en África oriental; según Brunet et al. (1995):

"Si el origen de los homínidos ocurrió rápidamente, seguido de rápida expansión, como parece probable, puede ser tan inútil buscar un centro de origen específico y localizado para los homínidos como lo es para cualquier otro grupo".

CRITERIO 3): el área del taxón que presenta las características más primitivas del grupo. El centro de origen se localizaría en la zona en la que se encuentren sus poblaciones de rasgos más primitivos (regla de progresión de Hennig). Este criterio implica que los taxones de rasgos más primitivos permanecerían en su área de origen, lo que no ha sido observado. La regla de progresión de Hennig es opuesta a la regla de dispersión de Darlington, Simpson, Brundin y Willis, según la cual los taxones primitivos se verían expulsados a áreas marginales por los taxones derivados, mientras que éstos se concentrarían en el área original (Figura 1). La regla de dispersión es más compatible con el criterio 1, aunque tampoco está apoyada en datos de observación concluyentes (Morrone \& Crisi, 1995).

Como alternativa ante la falta de convergencia de estos criterios aplicados a casos concretos, surge la escuela de Biogeografía Cladística. Los métodos de cladística de áreas no tratan de localizar el centro de origen de un grupo, sino que consideran el conjunto de las áreas de los descendientes como el área primitiva (ver un ejemplo en Lang, 1990). Este criterio implica que la diferenciación de las especies se produce por vicarianza (subdivisión del área de la especie ancestral), un ejemplo de lo cual podemos ver en García-París et al. (2000). Según la cladística de áreas, la dispersión fuera del área primitiva sería un fenómeno accidental, excepcional e impredecible (Platnick y Nelson, 1978; Wiley, 1988). Las posibles dispersiones se detectarían a posteriori, si los cladogramas de las mismas áreas obtenidos con distintos taxones resultan incongruentes. También pueden ser detectadas a priori cuando la evidencia geológica, la coexistencia de especies hermanas o la periferia del área de una especie eurícora señalan probables apocorías o dispersiones que modifican el área primitiva (Sanchiz, 1979).

Según otros procedimientos basados en la Biogeografía cladista, algunos autores tratan de localizar un área ancestral restringida de un taxón a partir de un cladograma de áreas. Utilizan para ello un criterio probabilístico de parsimonia, según el cual el área original sería la que requeriría menor número de eventos de dispersión y extinción de 

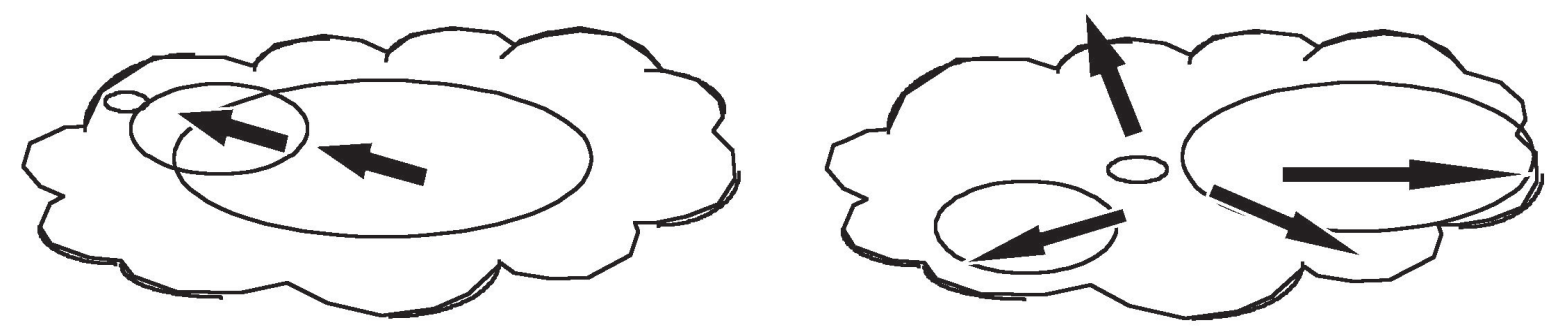

Fig. 1.- Modelos ideales de expansión de una especie de organismos utilizados para inferir su centro de origen. A la izquierda, modelo de dispersión de Darlington y Simpson, donde las poblaciones primitivas se verían rechazadas a áreas marginales y las poblaciones avanzadas se concentrarían en las proximidades del área ancestral, que mostraría una mayor diversidad. A la derecha, modelo opuesto de Hennig ("regla de progresión"), según la cual las poblaciones primitivas permanecerían próximas a su centro de origen y las poblaciones avanzadas se dispersarían a zonas alejadas. Los criterios para localizar del centro de origen conducen así a inferencias dispares.

Fig. 1.- Ideal models for the expansion of a species of organisms, used to infer its centre of origin. To the left, Darlington and Simpson's model of dispersion, where the primitive populations would be rejected to marginal areas and the derived populations would concentrate in the proximities of the ancestral area, which would show a higher diversity. To the right, the opposite Hennig's model ("progression rule"), according to which the primitive populations would remain near to their centre of origin and the derived populations would be dispersed to far away areas. Thus, approaches to locate the centre of origin lead to disparate inferences.

taxones (Bremer, 1992; Ronquist, 1994; Hausdorf, 1998; ver ejemplos en Repetur et al., 1997, o Voelker, 1999). Este criterio se revela inconsistente, pues equivale a la regla de progresión de Hennig si no hay paralogías (áreas con más de un taxón, o taxones en más de un área); y si las hay, el criterio señala como supuesta área ancestral el área más repetida o conflictiva, pero no el centro de origen del grupo al que no hay modo de localizar a partir de las áreas de los descendientes (Ebach, 1999).

Ante esta situación, podemos intentar asegurar al menos la pertinencia del concepto de centro de origen (o de área ancestral), aunque su detección no sea operativamente posible, utilizando el método hipotético-deductivo. Estudiaremos la aplicabilidad del concepto situándolo dentro de un modelo biogeográfico concreto, que permita extraer deducciones verificables. Si estas deducciones pueden ser confirmadas por observación, el concepto podrá considerarse pertinente al menos dentro del modelo concreto.

\section{El "modelo simétrico" de expansión de las especies}

El concepto de centro de origen se ha utilizado junto con el de dispersión (expansión del área de una especie fuera de sus límites), que es un postulado de la escuela de Biogeografía Histórica denominada Biogeografía dispersalista. Esta escuela está ejemplificada en Darwin y Wallace aunque es muy anterior, y en sus principios refleja nuestra propia historia humana cargada de invasiones e imperios colonizadores.

El concepto de centro de origen puede precisarse dentro de un modelo concreto de Biogeografía Histórica. Veamos cómo lo expresan Martín Piera \& Sanmartín (1999):

“...todavía muchos biogeógrafos siguen refiriéndose a este concepto darwiniano (sic) de forma despectiva, a pesar de que es indudable que la evolución tiene lugar en unas coordenadas de tiempo y espacio y por ello es muy improbable que un nuevo taxón aparezca al mismo tiempo a lo largo de todo el área del ancestro, por evolución simultánea y paralela. Antes al contrario, aparecerá en un área cuyas condiciones le sean particularmente favorables, a partir de la cual extenderá su rango de distribución geográfica a favor de las condiciones ambientales que le son favorables y/o de la propia dinámica de la corteza terrestre".

Según este uso del concepto de centro de origen, similar al que hacen muchos autores y cuya formulación se remonta al menos hasta Cuvier, la población primitiva de una especie se multiplicará y dispersará desde su área ancestral restringida, extendiéndose por un área previamente no ocupada por dicha especie. La expansión geográfica progresaría hasta llegar a un límite que le impediría seguir extendiéndose, al menos temporalmente, hasta que el área de distribución alcance un cierto equilibrio 

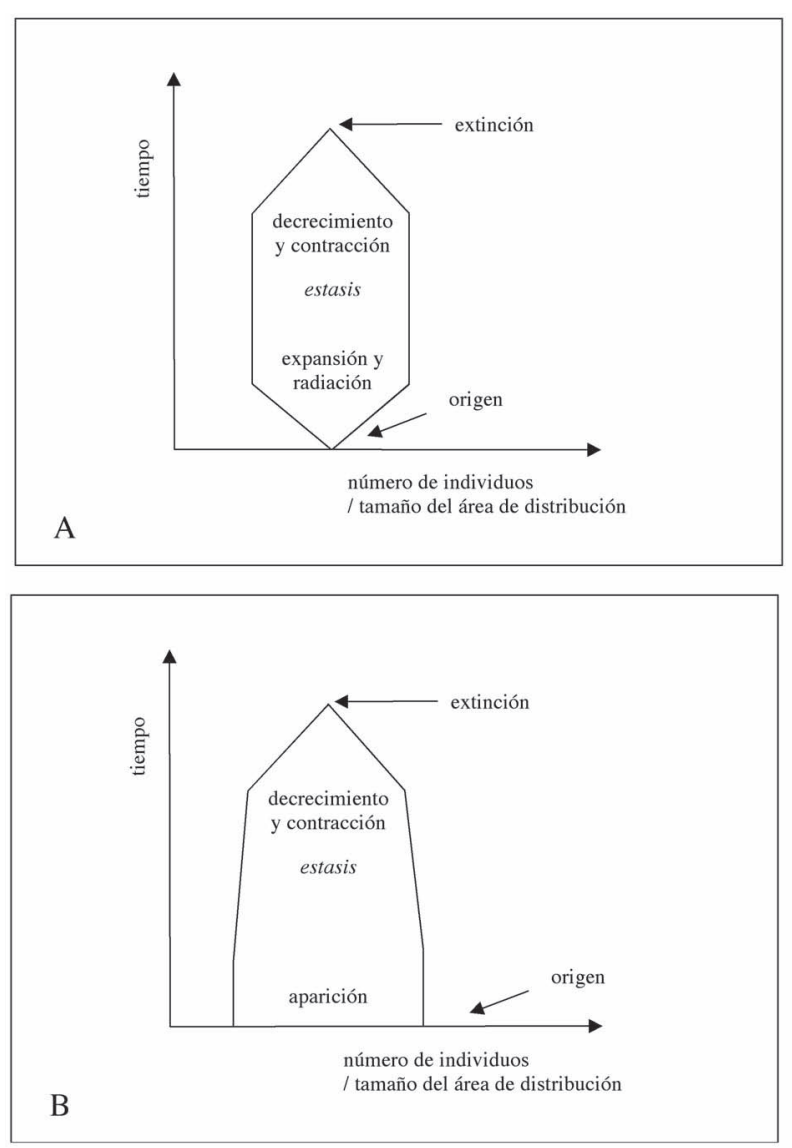

Fig. 2.- A) Modelo simétrico. Diagrama espacio-temporal de la existencia de una especie, según Jenkins (1994): los procesos de aparición-expansión del área y contracción-extinción se conciben aproximadamente simétricos en el tiempo. B) Modelo asimétrico. Representación del modelo alternativo propuesto en este trabajo. Los procesos de aparición y extinción se conciben como asimétricos en el tiempo, con una amplia extensión inicial del área y una contracción lenta, previa a la extinción.

Fig. 2.-A) Symmetric model. Spatio-temporal diagram of the existence of a species, according to Jenkins (1994): the processes of emergence-expansion of the area and contraction-extinction are considered approximately symmetrical in time. B) Asymmetric model. Representation of the alternative model proposed in this work. The emergence processes and extinction are considered as asymmetric in time, with a wide, extensive initial area and a slow contraction, previous to the extinction.

dinámico. Los factores que limitan la expansión se conciben generalmente como un tipo de barrera externa (ecológica o geográfica), tal como indica la formulación de Cuvier citada anteriormente. Según esto, las especies serían invasoras en gran parte de su área de distribución y sólo serían aborígenes en la pequeña zona restringida que constituyó su cen- tro de origen, si es que no lo han abandonado.

Este modelo puede servir de base para asegurar la pertinencia del concepto de centro de origen si se consiguen procedimientos de observación de algunas de sus implicaciones:

- La especiación (aparición de un taxón que ha adquirido una forma o modo de función distintas a otros) sería una singularidad local impredecible;

- Tras la especiación, se produciría generalmente la expansión del área de distribución, por dispersión de las poblaciones fuera del área ancestral;

- La expansión del área se vería favorecida o limitada por factores ambientales externos (aparición/desaparición de barreras);

Según este modelo, la dispersión fuera del área de distribución sería un proceso generalizado en todas las especies (por eso se ha denominado "escuela dispersalista" a quienes lo usan). En cuanto las condiciones les fueran favorables, las poblaciones tenderían a la expansión por una propiedad intrínseca suya, como la expansión de los gases. El proceso de expansión del área sería inherente a las especies, de modo análogo al crecimiento de un organismo controlado por factores internos, y al que los factores externos sólo pueden afectar incidentalmente o hacerlo inviable. Un ejemplo de la aplicación de este modelo se encuentra en la expansión postglacial de dos mil especies de plantas en Siberia a partir de un primitivo hipotético refugio local (Hultén, 1937 fide Hengeveld, 1990). Cada grupo de especies de estas plantas se expande ocupando "áreas equiformales progresivas" anidadas (las mayores incluyen a las menores en su interior). Según Hengeveld (1990, pg. 92):

"La dinámica de las áreas de distribución de especies sería, tal como la concibe Hultén, un proceso histórico que ocurre independientemente del clima, más que uno ecológico".

Las especies se expandirían por sus propios medios y a su propio ritmo y su expansión no estaría controlada por factores externos.

La expansión del área primitiva de una especie se concibe así como un proceso generalizado en todas las poblaciones originales, que no necesita de aporte de energía adicional, análogo al proceso adiabático de expansión de los gases. Las especies como los gases tendrían la propiedad de ocupar todo el espacio disponible en cada momento (se adaptarían al "recipiente"), sin que el proceso exija variación en su energía interna, intercambio de trabajo o aumento de entropía. La expansión de las 
especies sin embargo necesitaría tiempo para extenderse a través de la reproducción y de la dispersión, mientras que la de los gases no. En la Figura 2A se muestra este modelo en un diagrama tomado de Jenkins (1994), que representa el número de individuos (y consecuentemente la ocupación del espacio) durante la existencia de una especie, y concibe como simétricos en el tiempo los procesos de expansión inicial y contracción final del tamaño de las poblaciones (y por tanto del área ocupada por ellas). Distinguiremos este modelo de otros modelos concebibles dentro de la Biogeografía Histórica con el nombre de "modelo simétrico".

Para tratar de verificar la validez del modelo simétrico hemos de extraer de él deducciones que puedan ser observadas. Por ejemplo:

1) la aparición de una nueva especie sería un fenómeno local y singular, restringido espacial y temporalmente. El modelo simétrico se vería refutado si una especie apareciese simultáneamente en un área de distribución muy extensa, o si surgiera repetidamente en distintas ocasiones.

2) la expansión del área de una nueva especie procedería linealmente, a velocidad aproximadamente constante. El modelo simétrico se vería refutado si una nueva especie no expandiera su restringida área inicial, o si ésta se modificara de forma aleatoria.

Disponemos actualmente de nuevos medios de observar y documentar con datos actuales y paleontológicos las modificaciones del área de distribución de algunas especies, que podemos aplicar a la comprobación de estas dos implicaciones del modelo simétrico. Primero centraremos las observaciones sobre especies actuales y acudiremos posteriormente a datos paleontológicos.

\section{Observaciones sobre expansión del área de distribu- ción de especies actuales}

Para poder estudiar la dinámica del área original de una nueva especie actual, habríamos de disponer de observaciones directas sobre procesos de especiación, eventualmente seguidos de expansión, registrados ante un observador en algún lugar. Sin embargo no se ha documentado aún la formación y expansión de una especie nueva que haya aparecido en la naturaleza o en el laboratorio. La identificación de una especie desconocida (y la consecuente descripción de una "especie nueva"), que ocurre con cierta frecuencia en estudios de sistemática de algunos grupos, se interpreta unánimemente como el descubrimiento de una especie previamente existente, no como la reciente aparición de una nueva especie. Por eso las formas de especiación tipificadas (alopátrica, simpátrica, parapátrica, paralela, estasipátrica, etc.) no se han observado, sino que se han inferido en base a las pautas que muestran las diferencias entre poblaciones (Otte y Endler, 1989; Howard \& Berlocher, 1998).

En contraste, tenemos datos de la expansión del área de distribución de algunas especies previamente establecidas y particularmente observables, como las floraciones del plancton oceánico y las especies invasoras. Más tarde veremos un ejemplo del primer tipo, mientras que del segundo tipo conocemos numerosos casos.

\section{LA EXPANSIÓN DE ESPECIES INVASORAS}

Hay numerosos datos actuales sobre casos particulares de especies invasoras que están expandiendo rápidamente sus áreas de distribución. Se conocen muchos casos de dispersión de organismos exóticos que han producido invasiones a gran o pequeña escala en medios terrestres y acuáticos, tanto de especies individuales como de comunidades de varias especies. El tema despierta gran interés, y son numerosos los simposios y trabajos recientes (ver por ejemplo Mooney \& Cleland, 2001 y D'Antonio \& Kark, 2002). Se han documentado numerosos casos de invasiones debidos a acciones antrópicas accidentales o premeditadas, como introducciones de especies domésticas, comensales, de interés cinegético, parásitos, plagas de cultivos, epidemias, etc., y modificaciones de barreras geográficas (como las "migraciones lessepsianas" ocurridas tras la apertura del canal de Suez, ver Por, 1978). Son especialmente frecuentes las invasiones de regiones insulares por especies de regiones no insulares, con efectos generalmente letales para las especies autóctonas.

La expansión de especies invasoras se produce tras la desaparición brusca de una barrera y su dispersión es muy rápida (hasta varios kilómetros por día). Los procesos de invasión se han medido, observado y simulado usando modelos de difusión a los que a veces se añaden factores de crecimiento, densidad e interacciones locales. Las observaciones obtenidas en numerosos casos sobre la velocidad del proceso de expansión se ajustan a modelos lineales, tanto si el área se expande en forma de frente de onda como en forma de coalescencia de parches (ver Hengeveld, 1989; Andow et al., 1990; Van den Bosch et al., 1990; Kooijman, 1993; Lewis \& Pacala, 2000 y referencias citadas por 


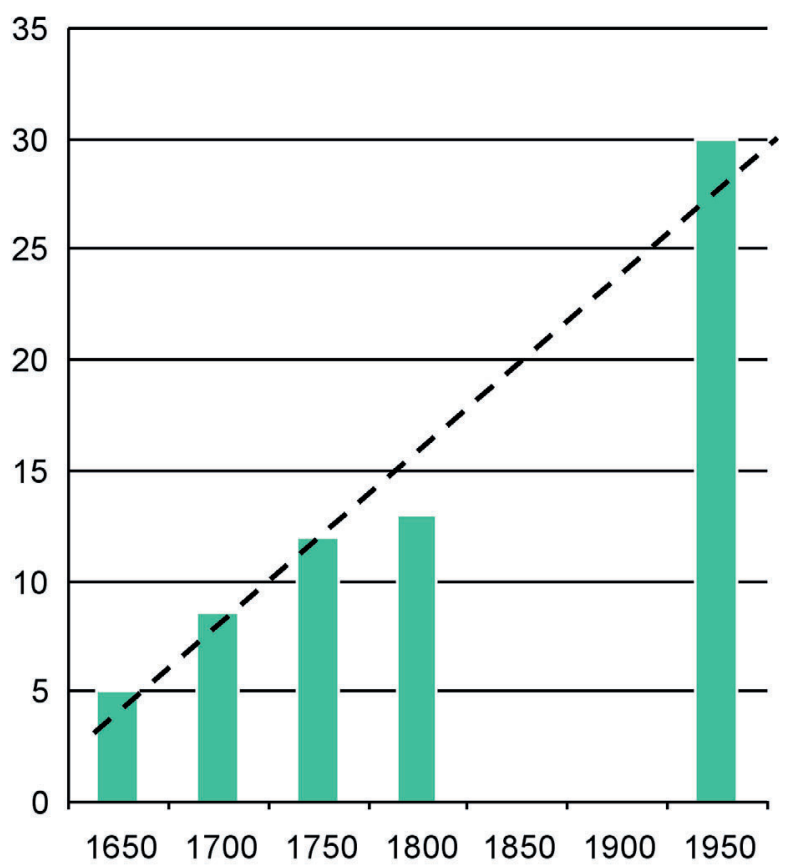

Fig. 3.- Crecimiento lineal del área sucesivamente ocupada por colonias de líquenes, que muestran tasas de crecimiento constante. La expansión del área de distribución de las especies invasoras también sigue un modelo de crecimiento lineal. Para que la expansión proceda a tasa constante, las poblaciones en el borde del área han de crecer exponencialmente, mientras que las del interior del área no contribuyen a la expansión y apenas crecen (Kooijman 1993,pg. 147 y referencias allí).

Fig. 3. - Lineal growth of the area successively occupied by lichen colonies showing constant growth rates. The expansion of the distributional area of invasive species also follow a lineal growth model. In order that the expansion take place in a constant rate, the populations in the borders of the area must grow exponentially, while those of the inner part of the area do not contribute to the expansion and hardly grow (Kooijman 1993, pg. 147 and references therein).

ellos). El ajuste de los modelos a funciones lineales de velocidad aproximadamente constante (Figura 3), indica que la expansión del área de especies invasoras es un proceso regulado por factores intrínsecos a las especies. La expansión del área de una especie invasora es un proceso análogo al del crecimiento sobre una superficie de un biofilm microbiano o liquénico, y el ajuste de ambos procesos a modelos matemáticos lineales implica su necesaria regulación por factores intrínsecos. Los procesos lineales de expansión en una superficie requieren coordinar un crecimiento exponencial de las poblaciones marginales y una inhibición simultánea del crecimiento de las poblaciones centrales, que apenas contribuyen a la expansión (Kooijman, 1993). La invasión del espacio disponible observada en un proceso lineal de crecimiento y expansión requiere un estricto ajuste de las tasas de reproducción y migración de las poblaciones en función de su posición espacial. Por tanto, los datos sobre especies invasoras verifican la deducción 2 del modelo simétrico: la expansión del área de las especies invasoras observadas se ajusta a un proceso lineal, regulado e intrínseco de las especies.

Como se documenta en el registro fósil, en el pasado también han ocurrido sin intervención humana rápidas invasiones de especies exóticas carentes de posibles antecesores en la región invadida. A estos dispersalistas a menudo se les denomina impropiamente inmigrantes, ya que la migración implica desplazamiento dentro del área de distribución de la especie. Un ejemplo de aparición repentina de especies exóticas es el de los équidos del Viejo Mundo (África, Europa, Asia y el subcontinente indio). El caballo (Equus) hace unos 2,5 millones de años (Ma) sustituyó rápidamente en toda el área a Hipparion, el cual había sustituido rápidamente hace unos $11 \mathrm{Ma}$ a Anchitherium que habitaba Eurasia durante los 7 Ma anteriores tras una invasión igualmente repentina. Estas apariciones constituyen marcados y útiles horizontes biocronológicos prácticamente simultáneos, atribuidos a la desaparición periódica de la barrera del estrecho de Behring entre Norteamérica, donde abundaban los équidos, y el Viejo Mundo, donde no proliferaban. Sin embargo los taxones exóticos llegados al Viejo Mundo no se registran previamente en el área de procedencia inferida, ni se ha conseguido documentar un proceso de expansión gradual, sino al contrario, como las apariciones de otras muchas especies-guía, resultan "geológicamente instantáneas" (ver más abajo). Otras especies norteamericanas no aprovecharon la conexión, y cada aparición fue acompañada de pocos cambios.

Estas apariciones de especies particulares difieren de los eventos de renovaciones masivas de comunidades formadas por numerosas especies, que en ocasiones se detectan en el registro fósil acompañadas de notables cambios ambientales como p. ej. la expansión postglacial de las plantas siberianas de Hultén que vimos anteriormente. Este tipo de expansión implica a todo el ecosistema, y no a una o unas pocas especies. En ambos casos, la procedencia de la mayoría de las especies exóticas o invasoras que se detectan en el 
registro fósil resulta imprecisa y suele ser motivo de controversia (ver p. ej. el caso de los mamíferos del Hemisferio Norte al principio del Eoceno: Gingerich, 2000; Krause \& Maas, 1990, o los de la Península Ibérica durante el Mioceno: Pickford y Morales, 1994; Van der Made, 1999). Por esta razón Olson (1971) se quejaba de que el origen de todas las especies siempre parecía estar "en otro lugar" como vimos antes. Más de 30 años después, no hemos conseguido aún precisar el centro de origen de la mayoría de especies conocidas, incluidas las especies domésticas.

Dadas las frecuentes apariciones y desapariciones de barreras geográficas y las numerosas ocasiones que han existido para la dispersión de las especies, las invasiones o expansiones podrían haber homogeneizado hace tiempo las regiones biogeográficas del planeta. El hecho de que esto no haya ocurrido indica que las biotas son resistentes a las introducciones y que éstas sólo ocurren en casos particulares. Las características especiales que presenta la dispersión de taxones invasores incluyen: 1) la desaparición de la barrera que limitaba su área de distribución; 2) la procedencia de los colonizadores desde áreas-fuente no aisladas, donde las especies invasoras han sufrido previamente numerosas interacciones con otros taxones; y 3 ) la introducción en un ecosistema donde encuentren un vacío ecológico. Veremos que las especies endémicas procedentes de áreas restringidas no se comportan de la misma manera.

\section{LOS ENDEMISMOS Y LA ESPECIACIÓN INSULAR}

Conocemos numerosos casos de especies restringidas a un área muy limitada (endemismos), muchos de ellos propios de áreas insulares sensu lato (espacios delimitados, diferenciados de su entorno y de extensión muy reducida respecto a la región circundante). En oposición a las especies eurícoras que ocupan una amplia área, las especies endémicas o estenócoras en caso de expandirse nos proporcionarán modelos biogeodinámicos en los que su centro de origen estaría localizado. Por ello el estudio de los procesos de modificación de las áreas de especies endémicas es de gran interés para la Biogeografía Histórica.

Las especies endémicas pueden ser interpretadas como paleoendemismos (especies refugiadas en hábitats relictos) o como neoendemismos (especiación local reciente aún no expandida). El modelo simétrico predice que una especie endémica, sobre todo si es neoendémica, mostrará tendencia a dispersarse fuera de su área y a expandirse regularmente en el momento en que las barreras desaparezcan.
Significativamente no se conocen casos de especies endémicas que se hayan convertido en invasoras o que tiendan a expandirse a otras regiones, a pesar de numerosos experimentos de aclimatación en áreas favorables, intensamente ensayados sobre todo en especies amenazadas de extinción. Frecuentemente ocurre al contrario, las especies endémicas tienden a extinguirse cuando interaccionan con especies más cosmopolitas, y su supervivencia se relaciona a menudo con la existencia de zonas protegidas (refugios). La falta de capacidad de expansión de la gran mayoría de especies endémicas es opuesta a las predicciones del modelo simétrico.

Ejemplos bien estudiados lo constituyen los endemismos insulares, que han atraído la atención de numerosos autores por su asombrosa disparidad de formas o su riqueza en especies. Entre ellos hay ejemplos clásicos en Biología Evolutiva, como los pinzones de las Islas Galápagos, las moscas Drosophyla de las Islas Hawai o los peces cíclidos del Lago Victoria. Muchos de estos singulares taxones han desaparecido o están gravemente amenazados en vez de haber conseguido expandirse ningunos de ellos.

Los datos sobre especies endémicas actuales se complementan con numerosos datos paleontológicos de especies insulares, que corroboran que los extraordinarios endemismos abundantemente registrados en islas no se comportan nunca como especies en expansión. Es el caso por ejemplo de las 25 especies endémicas de mamíferos terrestres de las grandes islas del Mediterráneo, diferenciadas durante los últimos 6 $\mathrm{Ma}$, y extinguidas durante el Holoceno, al contacto con las especies invasoras del continente que acompañaron al hombre (Cheylan, 1991; Alcover et al., 1998; Moyá-Solá et al., 1999). Ejemplos de vertebrados endémicos extinguidos documentados en nuestro país encontramos en las Islas Baleares (Baleaphryne, Nesiotites, Carbomys, Margaritamys, Eivissia, Hypnomys, Gymnesicolagus, Alilepus, Myotragus) y en las Islas Canarias (Gallotia, Canariomys, Malpaisomys), pero se pueden citar muchos otros, como el asombroso hombre endémico del Paleolítico extinguido en Cerdeña (Spoor y Sondaar, 1986).

Aunque el fenómeno de la incapacidad colonizadora de las especies endémicas debe ser aún corroborado con muchos más datos, es significativo que algunos raros casos de supuestos endemismos excepcionalmente expansivos han sido refutados. Por ejemplo es paradigmático el caso del muflón (Ovis musimon), cuyas poblaciones procedentes de las islas de Córcega, Cerdeña y Chipre han sido introducidas en el continente como especie de interés cinegético y se han adaptado con éxito. Sin 
embargo, en contra de lo comúnmente admitido, no se trata de un caso de expansión de una especie endémica, pues esta especie existía en el continente europeo durante el Pleistoceno y estaba ausente en las islas; por tanto su comportamiento expansivo se relaciona precisamente con una extensa área de procedencia original (su centro de origen, como es habitual, es impreciso; ver Uerpmann, 1987).

Las islas por tanto nunca muestran ser centros de origen de especies dispersalistas, que podrían aprovechar conexiones favorables entre áreas para ampliar su distribución, sino que al contrario son sumideros de las nuevas especies que se originan en ellas a partir de las poblaciones colonizadoras procedentes del área-fuente. Contra la predicción del modelo, en muchas especies neoendémicas bien documentadas, de las que podemos precisar su centro de origen, la aparición local no va seguida de expansión.

Los estudios sobre endemismos insulares proporcionan una refutación adicional del modelo simétrico. La especiación insular muestra pautas generales, como la tendencia a reducir el número de crías, a modificar el tamaño de macro- y micromamíferos hacia tamaños intermedios, o a perder capacidad de vuelo en aves e insectos. Además de estos procesos macroevolutivos, se ha detectado en archipiélagos un proceso biogeográfico histórico descrito con el nombre de "ciclo del taxón" (Wilson, 1961; Ricklefs y Cox, 1972). Según este modelo, descrito con datos morfológicos en hormigas, lagartos y aves y recientemente confirmado con datos moleculares, las especies que colonizan un archipiélago desde el área fuente pasan por varias fases: 1) las especies recién llegadas poseen una amplia distribución geográfica en numerosas islas y escasa diferenciación de sus poblaciones, 2-3) y pasarían sucesivamente a restringir su distribución a medida que se van diferenciando, hasta 4) reducir su área a una única isla y presentar la máxima diferenciación, lo que precede a su extinción. El ciclo se repite cuando nuevas especies invaden el archipiélago, lo que convierte a las áreas-fuente en determinantes del inicio del ciclo. Las regularidades observadas sobre los tiempos estimados de diferenciación y de contracción de las áreas de distribución, sugieren que estos procesos no son controlados por factores ambientales sino que son intrínsecos a las especies (Ricklefs y Bermingham, 2002). Estos autores estiman la duración del ciclo del taxón alrededor de $1 \mathrm{Ma}$ en las Antillas, independientemente del grupo de organismos estudiados y de los ciclos climáticos del Pleistoceno.
Aunque el ciclo del taxón se ha descrito en áreas insulares, su pauta puede encontrarse igualmente en endemismos de áreas no insulares, como p.ej. el modelo de especiación de las salamandras tropicales citado anteriormente (García-París et al., 2000)

La pauta que muestra el ciclo del taxón se opone a la esperada por el modelo simétrico. En vez de relacionarse con una expansión, la diferenciación de los taxones (especiación) va asociada al proceso de contracción del área de distribución. En contraste con el modelo simétrico de la historia de un taxón representado por Jenkins (1994, Figura 2A), la ocupación del área durante el ciclo del taxón es asimétrica, iniciándose con una extensión máxima de la especie colonizadora del área insular y reduciéndose gradualmente hasta su extinción (Figura 2B). Este modelo asimétrico de desarrollo del área de un taxón se presenta en este trabajo como propuesta alternativa al modelo de Jenkins (1994), y se corrobora con datos paleontológicos como muestra en su trabajo el propio Jenkins (1994) de distribuciones de numerosas especies de foraminíferos planctónicos asimétricas en el tiempo. Ambos modelos simétrico y asimétrico se muestran simplificados en los diagramas la Figura 2 , y en ellos no se indican las diversas modificaciones del área que se puedan producir a lo largo de la duración de un taxón.

En resumen, en los raros casos de especies insulares neoendémicas en que podemos conocer con precisión el centro de origen de una especie, no se observa la esperada expansión. La diferenciación de especies autóctonas en islas ha sido frecuentemente documentada, de modo que su carácter de neoendemismos autóctonos locales está en muchos casos garantizado. El centro de origen de muchas especies insulares es la isla misma, donde podemos seguir a veces la pauta de transformación de la especie ancestral que en ocasiones coincide con una especie presente en el área-fuente. Los eventos de recolonización muestran que hay ocasiones favorables para el intercambio de especies entre el área-fuente y las áreas insulares. Pero esta difusión resulta ser unidireccional y se produce en sentido contrario a la predicción: la transferencia de poblaciones viables se produce del área-fuente al áreasumidero insular, como describe el "ciclo del taxón", y no se conocen casos de invasiones en sentido contrario. La diferenciación local se acompaña de la restricción del área de la nueva especie, en vez de radiar en todas direcciones. Todos los casos documentados muestran estabilidad o extinción del neoendemismo, en contra de lo esperado por el modelo simétrico que predice su expansión. 
Por tanto, en los endemismos insulares en que el centro de origen de un taxón puede ser inferido, resulta que el área ancestral del taxón es al mismo tiempo irónicamente su mayor y más amplia área de distribución, e incluye asimismo lo que será su lugar de extinción. Tanto el ciclo del taxón de Wilson como los documentos paleontológicos muestran que en las especies insulares, el centro de origen o área ancestral no se identifica en un área restringida desde donde se expande la nueva especie, sino en el área más o menos amplia que ya ocupa el taxón inicialmente, y que se irá contrayendo hasta la extinción del taxón. En las islas, el desarrollo de los taxones se ajusta al modelo asimétrico de la Figura 2B, refutándose el modelo simétrico.

Se ha de estudiar por tanto si el desarrollo de los taxones en regiones no aisladas puede ser similar al de las islas, o si por el contrario las islas son un caso peculiar y particular del comportamiento de las nuevas especies. Para ello, veremos otros casos basados en datos paleontológicos, en los que los eventos de aparición y extinción de especies-guía y la historia paleobiogeográfica de algunos taxones permiten estudiar la aplicabilidad del modelo simétrico y del concepto de centro de origen.

\section{Observaciones sobre aparición y expansión de espe- cies en el registro fósil}

Los datos documentados en el registro fósil nos permiten el seguimiento de los procesos de origen y expansión de especies nuevas, y podremos así verificar si su aparición es local o regional y si su expansión es o no un proceso lineal. Trataremos primero los casos de aparición y expansión de especies-guías, que son taxones de interés bioestratigráfico utilizados en la datación geocronológica, y posteriormente veremos ejemplos de historia biogeográfica de algunos taxones ibéricos.

\section{LA APARICIÓN DE ESPECIES-GUÍA}

Los datos paleontológicos son por el momento los únicos que permiten documentar las pautas temporales de aparición de nuevas especies, a falta de observaciones directas sobre procesos actuales de especiación. Los grupos de fósiles mejor representados y estudiados cronológicamente son los utilizados en bioestratigrafía (cocolitofóridos, foraminíferos, ammonites, graptolitos, conodontos, mamíferos, etc.), por su amplia distribución y rápida evolución. En los casos de linajes supervivientes en la actualidad, como es el caso de los cocolitofóridos, los foraminíferos y los mamíferos, podemos comprobar que la distinción de especies en base a fósiles es comparable con la realizada sobre ejemplares recientes.

En todo el mundo o en amplias regiones se registran similares sucesiones bioestratigráficas de numerosas especies de estos grupos de fósiles. Entre estas especies se seleccionan las especiesguía utilizadas para subdividir la sucesión en biozonas. Cuando la misma sucesión de especies-guía puede generalizarse a escala regional o global, se seleccionan eventos de referencia (aparición, predominio, coexistencia o extinción de especiesguía), considerados como horizontes sincrónicos (datum biocronológico, Figura 4). Se definen así las escalas biocronoestratigráficas generales para subdividir el tiempo geológico (dataciones relativas, Figura 4).

La gran mayoría de estos horizontes escogidos se definen por eventos de aparición de nuevas especies-guía, aproximadamente simultáneos a la escala de resolución temporal disponible (Paul, 1987). Sólo en muy raras ocasiones se definen horizontes biocronológicos en base a otros eventos paleobiológicos, como la extinción o la asociación de especies-guía. La asimetría existente entre los numerosos horizontes de aparición y los escasos horizontes de extinción de especies-guía, refleja la asimetría documentada en el registro fósil entre el breve tiempo del proceso de aparición y el largo tiempo del proceso de extinción de las especies-guía. Esa asimetría está representada en la Figura 2B.

La desaparición de una especie es frecuentemente diacrónica en distintas regiones, por lo que es muy difícil precisar el momento de su extinción o desaparición de la más reciente de sus poblaciones (Jenkins, 1994). Aún actualmente aparecen especies que se creían extintas, lo que corrobora que la extinción está precedida por un lento proceso de desaparición diacrónica de poblaciones, que ocurre en diferentes momentos en distintas localidades de su área. Según Jenkins (1992), la desaparición diacrónica de poblaciones y reducción escalonada del área previa a la extinción es una pauta generalizada en todas las especies que puede utilizarse para predecir su extinción antes de que ocurra. A la inversa, la sincronicidad de los horizontes de aparición de las especies-guía se ve confirmada con procedimientos de datación y correlación que van mejorando la precisión de la escala geocronométrica (dataciones absolutas, Figura 4). Hasta donde los métodos geocronométricos permiten precisar, el fenómeno de aparición de una especie-guía es cuasi-sincrónico incluso a muy gran escala espacial. 


\section{transición Cretácico-Terciario}

\section{Cronología}

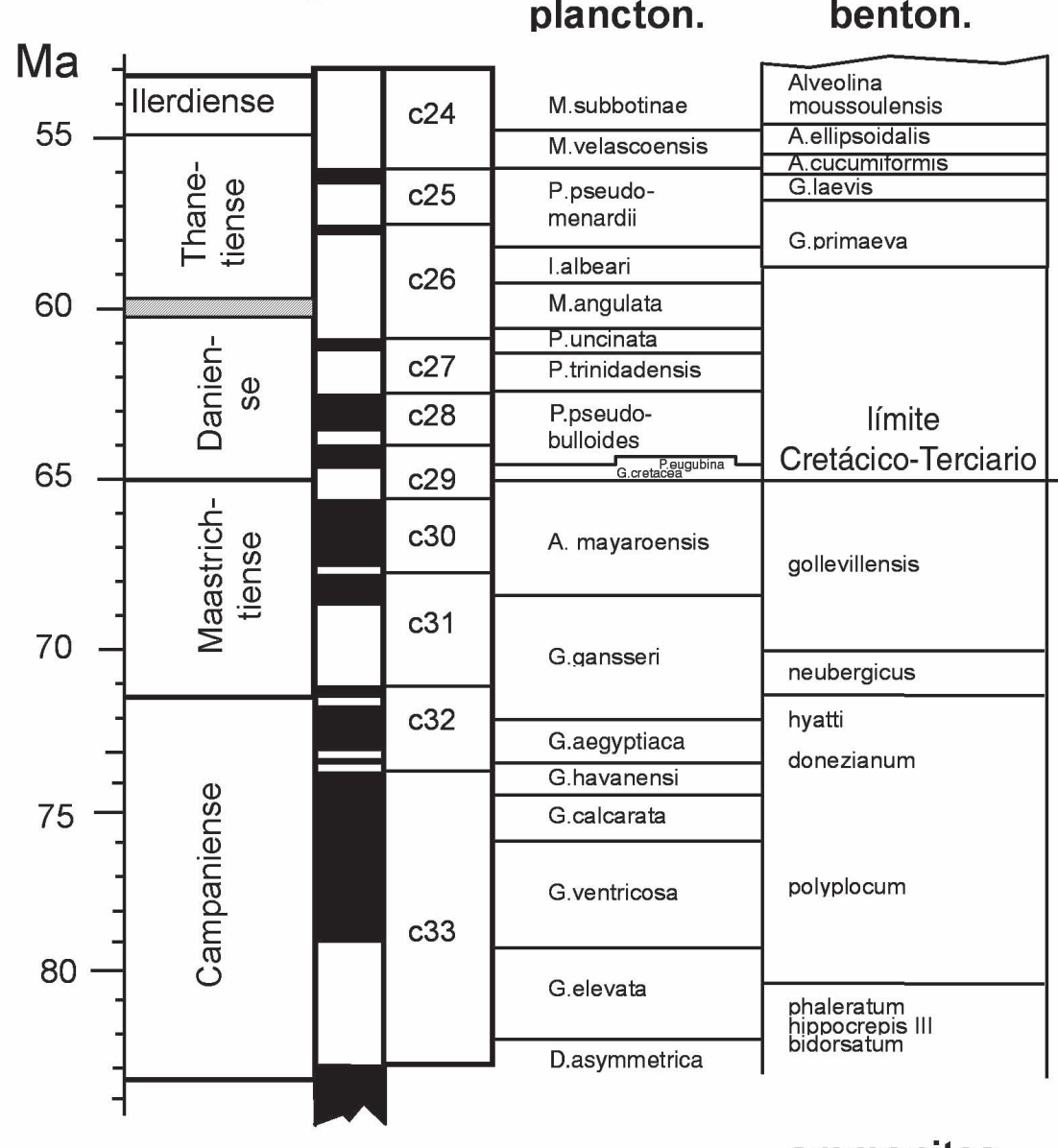

carofitas

Peckichara toscarensis

P.llobregatensis

Sphaerochara

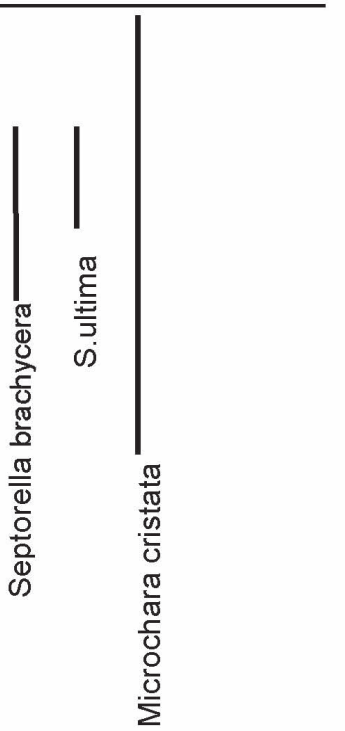

Fig. 4. - Subdivisiones del tránsito Cretácico-Terciario en base a eventos de aparición de especies-guía de foraminíferos planctónicos, ammonoideos y macroforaminíferos bentónicos. A la izquierda, la escala geocronométrica indica su edad en millones de años. A la derecha, aparición y duración de algunas especies de algas carofitas.

Fig. 4.- Subdivisions of the Cretaceous-Tertiary transition period based on events of guide-species appearance of planktic foraminifera, ammonoids and benthic foraminifera. At the left, the geochronometric scale shows the age in million of years. At the right, appearance and duration of some species of charophyte algae.

En la Figura 4 se muestran varias subdivisiones temporales de la transición entre las eras Secundaria y Terciaria, basadas en la aparición de nuevas especies de foraminíferos y ammonites de aplicación a escala global, en donde también se indica la aparición y la duración de algunas especies de algas carofitas de amplitud regional. Estas escalas han sido calibradas respecto a la escala geocronométrica por medio de la escala magnetoestratigráfica, que nos indica los periodos de magnetismo terrestre normal (en negro, campo magnético actual) e inverso (en blanco, campo magnético opuesto al actual). Los periodos de reversión magnética se consideran muy rápidos, y su relación con los eventos de aparición 
de especies-guía muestran que éstos son igualmente muy rápidos, de modo que ambos se consideran horizontes aproximadamente sincrónicos. En ambos casos, el término 'rápido' es relativo a la diferencia de 2 a 4 órdenes de magnitud entre la duración estimada del evento (horizonte de aparición de especies-guía, reversión magnética) y la del periodo (intervalo de estabilidad de las especies-guía y de la polaridad magnética).

En algunos casos se describen linajes anagenéticos, cuando una especie-guía puede considerarse transformación morfológica de una especie precedente, a la que reemplaza en toda su área de distribución (cronoespecies sucesivas, ver más abajo el caso del linaje Microtus brecciensis - M. cabrerae). Se corrobora esta hipótesis de transformación cuando las especies ancestral y descendiente no coexisten en ningún lugar. Al igual que el emplazamiento de especies-guía sin antecesores en la zona, el reemplazamiento de cronoespecies también resulta aparentemente simultáneo en toda el área de distribución anteriormente ocupada por la especie ancestral. Esta pauta de sucesión de especies descrita en los linajes anagenéticos es justo lo que el modelo simétrico excluye. La transformación prácticamente simultánea de una especie ancestral en una nueva especie descendiente a lo largo de toda su área de distribución se opone al modelo simétrico de especiación local y subsiguiente expansión lineal.

En otros casos, el registro fósil muestra la aparición en un mismo periodo de múltiples especies emparentadas (linajes cladogenéticos). En estos casos se suele aplicar por defecto el modelo simétrico, aunque no haya aún modo de corroborarlo; se asume que la especiación ocurrió 'en algún otro lugar' a partir de un antecesor supuesto o desconocido, desde donde las nuevas especies descendientes se expandirían linealmente.

Se han descrito centenares de horizontes de aparición de especies-guía en áreas muy extensas, que se consideran como eventos muy rápidos (sincrónicos para todos los efectos) separados por periodos de estabilidad. En las épocas con mayor número de horizontes se registran aproximadamente uno por cada 300.000 años (Ordovícico, Devónico, Jurásico); en las de menor número, uno cada dos o tres millones de años (Triásico, Cretácico) y en la mayoría de épocas se registra un promedio de un horizonte por cada millón de años. La duración de las especies-guía es mayor que estos periodos en la mayoría de casos, salvo en algunos raros casos en que coincide la aparición de una nueva especie-guía con la extinción de la especie-guía precedente. De los centenares de biohorizontes definidos, la pro- porción de los definidos por la extinción respecto a los definidos por la aparición de especies-guía es muy baja, pues como hemos dicho se comprueba en muchos casos que la desaparición de las poblaciones de una especie es diacrónica. La asimetría del registro de las especies-guía manifiesta claramente el contraste asimétrico entre:

1) el rápido fenómeno de aparición de una especie nueva en un área muy extensa, $\mathrm{y}$

2) el lento proceso de reducción de su área y desaparición diacrónica de sus poblaciones, hasta llegar a la extinción final que puede demorarse $\mathrm{y}$ ser difícil de asegurar (Figura 2B).

Por tanto, las especies-guía en el registro fósil aparecen en la mayoría de casos como un evento cuasi-sincrónico a escala regional cuya duración es más breve que lo que permiten medir los procedimientos geocronométricos. Por tanto, se considera para todos los efectos como un fenómeno simultáneo en toda el área y se utiliza como un horizonte biocronológico. Estos rápidos procesos de emplazamiento de nuevas especies se registran igualmente en casos de reemplazamiento anagenético de cronoespecies y en casos de cladogénesis.

Desde Darwin, la aparición repentina de las especies-guía (y de otras muchas especies) en el registro fósil se había atribuido a su tópica imperfección, pero ha sido repetidamente corroborada hasta ser integrada en el marco de la teoría puntuacionista de evolución de Eldredge y Gould. Estos autores han propuesto una explicación basada justamente en el modelo simétrico de Biogeografía Histórica. Como las escalas temporales de los procesos biológicos y de los geológicos son muy diferentes, se puede imaginar que la expansión gradual del área de una especie nueva que durase varios miles de años a partir de un pequeño centro de origen local sería difícilmente detectable en el registro geológico. Es sin embargo significativo que tampoco se detecte en la biosfera actual, lo que sugiere rápidos procesos de bifurcación.

Además, aunque la expansión súbita y generalizada de una especie nueva en una amplia área puede parecer biológicamente improbable, encontramos un caso análogo actual en la floración de Emiliania huxleyi (Lohmann 1902), un organismo unicelular del fitoplancton oceánico. Disponemos actualmente de un procedimiento casi ideal de observación vía satélite de la dinámica biogeográfica de esta especie, con la monitorización y registro de su actividad biológica lo más continuo y regular posible. Los movimientos de Emiliania huxleyi pueden ser observados en tiempo real por satélite debido a la coloración lecho- 
sa que produce en el mar cuando aparece. Sus poblaciones forman "blooms" o floraciones estacionales que cubren millones de kilómetros cuadrados y secuestran grandes cantidades de carbono en sus esqueletos calcáreos, lo que tiene efectos globales sobre el clima planetario. Desde los años 90 se ha puesto en marcha un proyecto internacional para seguir el desarrollo de este organismo, que ejemplifica el efecto de los organismos sobre el medio físico a escala global (Westbroek, 1991). La cantidad y calidad de información recogida en este proyecto no tiene parangón con ningún otro caso, y sus progresos pueden consultarse regularmente en su página web (http://www.soes.soton.ac.uk/staff/tt/eh/index.html).

La aparición de los blooms de Emiliania huxleyi ocurre cíclicamente en distintos puntos del globo. En el momento de la floración, la población de células con sus complejos esqueletos calcáreos surge simultáneamente en una gran extensión, que puede alcanzar más de $10^{5} \mathrm{~km}^{2}$, sin tiempo para la difusión de individuos por multiplicación y dispersión (Brown \& Yoder, 1994). En palabras de Brown (1998):

“...los satélites nos muestran que los blooms se desarrollan simultáneamente sobre una amplia área. Su despliegue es mucho más rápido de lo que sería posible si los blooms estuvieran causados por un 'efecto semilla' en que las células iniciales del bloom hubieran sido fisicamente transportadas desde otro lugar para iniciar el bloom".

Por tanto el inicio del bloom de Emiliania no es un fenómeno local y se comporta por el contrario como un gas que ocupa instantáneamente todo el volumen disponible, o como la floración de una especie vegetal en primavera, sin que parta de un centro de origen determinado y sin proceso lineal de expansión.

El caso de Emiliania huxleyi no es propiamente un fenómeno de especiación, sino de nueva aparición de una especie preexistente en un área despoblada. Sólo como analogía es comparable a los procesos de expansión de nuevas especies, y de momento es el único caso que permite observaciones continuas en tiempo real de la dinámica del área de distribución de una especie. $\mathrm{Su}$ documentación es muy reciente, de modo que sus procesos aún no han sido explicados y han de conocerse todavía datos sobre los factores que inciden en su distribución. Aunque los resultados no pueden ser directamente comparados con los de ninguna otra especie, para las que no existen de momento medios de observación de esta calidad, esta dinámica biogeográfica repetidamente observada no se ajusta a las predicciones del modelo simétrico. La aparición simultánea de la especie en un área de enorme extensión refuta la deducción 1 (la aparición no corresponde a un fenómeno local, sino regional) y la 2 (la expansión no procede linealmente). Brown (1998, ver más arriba) llama "efecto semilla" a la esperable aparición local y expansión progresiva requeridas por el modelo simétrico, que no se observa en este caso. Como vemos, la repentina aparición de Emiliania huxleyi simultáneamente en una extensa área es un fenómeno biológico análogo al que sugiere el fenómeno geológico de la aparición de especies-guía en el registro fósil y proporciona un ejemplo actual de una escala temporal comparable a la que sugieren los ejemplos del pasado.

\section{Historia PALEOBIOGEOGRÁFICA DE DOS TAXONES IBÉRICOS}

En algunos linajes paleontológicamente bien documentados es posible trazar su historia paleobiogeográfica, que permite el seguimiento parcial de las modificaciones del área de distribución de las especies en el pasado. Resumimos dos casos estudiados por nosotros y ligados a la Península Ibérica, los mamíferos Prolagus Pomel (Lagomorpha, Ochotonidae) y Microtus (Iberomys) Chaline (Rodentia, Arvicolidae).

El género Prolagus dura en Europa más de 20 Ma y comprende unas 22 especies, como se detalla en López-Martínez (2001). Se trata de un pequeño lagomorfo peculiar, de aspecto general similar a las actuales pikas (Ochotona), que estuvo representado por numerosas poblaciones muy ricas en individuos en paleoclimas cálidos y yacimientos de ambientes boscosos y húmedos. Este taxón se origina tras la transformación anagenética de Piezodus, un ochotónido que aparece repentinamente en Europa a finales del Oligoceno y al que se supone inmigrante desde Asia, donde no se ha registrado pero donde abundan los miembros del orden Lagomorpha. La historia paleobiogeográfica de Prolagus se resume en la Figura 5, donde se superponen las áreas de distribución ocupadas por todas sus especies durante tres periodos, Mioceno (entre 22 y $5 \mathrm{Ma}$ ), Plio-Pleistoceno (entre $5 \mathrm{Ma}$ y hace 10.000 años) y Holoceno (desde hace 10.000 años hasta la época actual). Durante el Mioceno el área de Prolagus alcanza su mayor expansión y se extiende desde la Península Ibérica hasta Europa Oriental y Anatolia. Su pauta de distribución es marcadamente latitudinal: su frecuencia, inicialmente mayor en el Norte de Europa, se traslada hacia el Sur a medida que el cinturón tropical se estrecha y Europa deriva hacia el Norte. Su diversidad sufre una importante crisis a finales del 


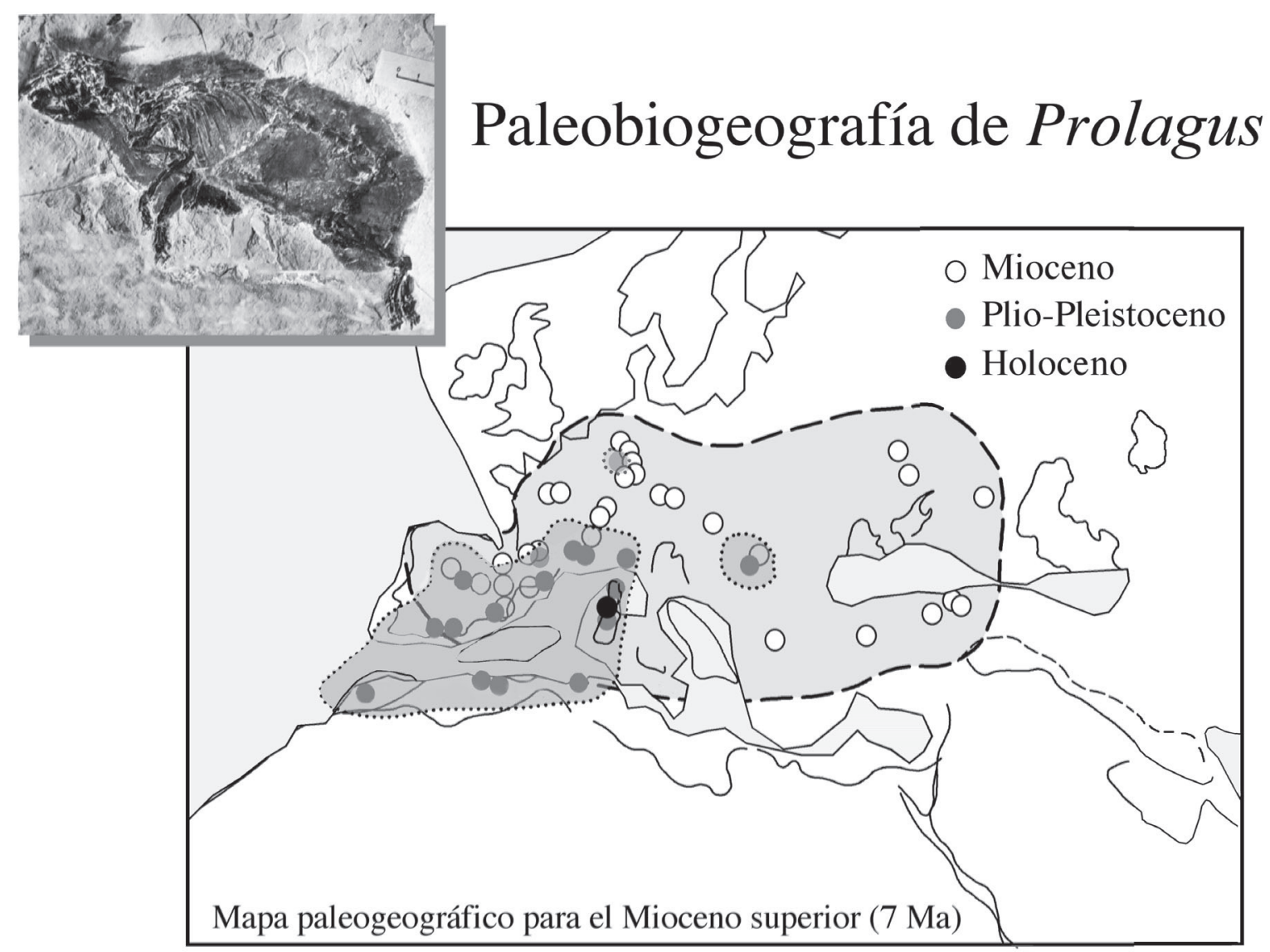

Fig. 5.- Contracción del área de distribución del mamífero lagomorfo Prolagus en Europa, desde su aparición hace 22 millones de años (Mioceno inferior) hasta su reciente extinción. Modificado de López-Martínez (2001).

Fig. 5.- Contraction of the area of distribution of the lagomorph mammal Prolagus in Europe, from its appearance 22 million years ago (early Miocene) until its recent extinction. Modified from López-Martínez (2001).

Mioceno Medio que afectó también a otros ocotónidos, tras la cual sólo sobrevive una especie de Prolagus, pero aparentemente sin reducción del área de distribución. A finales del Mioceno Prolagus coloniza África del Norte pero esta invasión no supone una expansión sino un desplazamiento, porque en conjunto su área se va reduciendo y fragmentando durante el Plio-Pleistoceno a medida que se diferencia en especies distintas, hasta que sólo persiste en Córcega y Cerdeña durante el Holoceno (Figura 5). Este proceso de fragmentación y reducción del área anuncia la futura extinción, tal como apunta Jenkins (1992, 1994), que aún tardó en llegar varios millones de años. Esta larga persistencia como paleoendemismo se debió a su aislamiento en estas islas-refugio, donde sobrevivió a las glaciaciones con poblacio- nes extremadamente abundantes. La entrada de poblaciones humanas y su fauna comensal le hicieron fuerte presión y finalmente se extinguió hacia el siglo XVII, tras quedar reducida su área de distribución al islote de Tavolara.

La historia paleogeográfica de Prolagus por tanto permite seguir con precisión el proceso de su extinción, pero no el de su origen y expansión que parece ya conseguido desde su inicio en el Mioceno inferior (cf. modelo asimétrico de la Figura 2B). Veremos un caso más reciente, que se desarrolla enteramente en el Cuaternario, por lo que el sesgo de información debido a la diferencia de edad de los yacimientos iniciales y finales es menor.

Microtus (Iberomys) es un topillo (roedor arvicólido) característico del Cuaternario del SW de Europa, que aún sobrevive precariamente en 


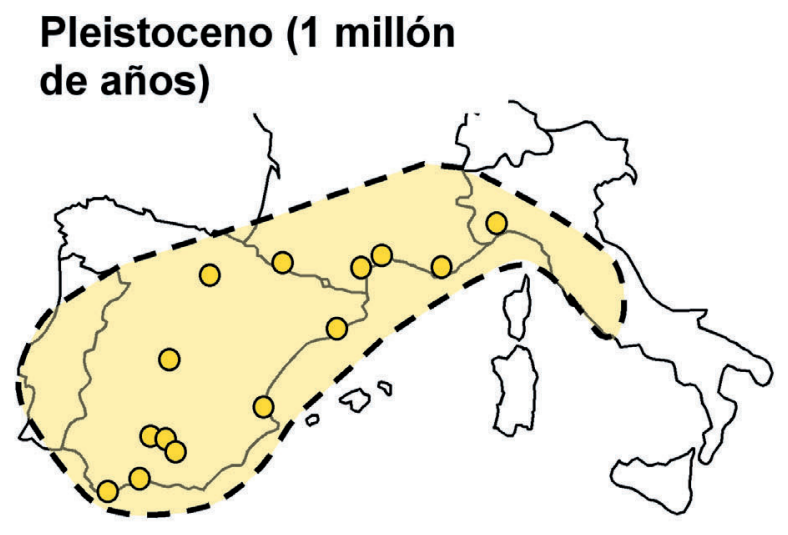

Holoceno (6.000 años)

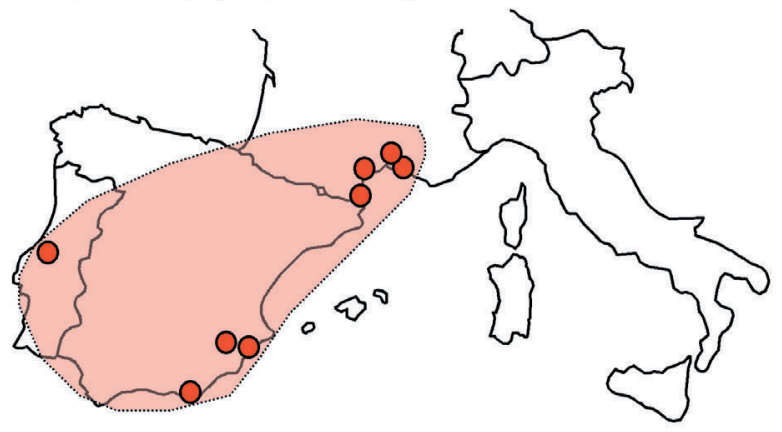

reciente

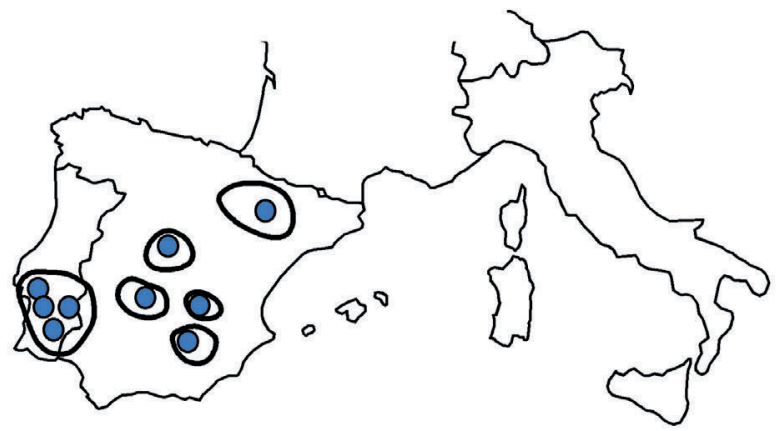

Fig. 6.- Modificaciones del área de distribución del roedor arvicólido Microtus (Iberomys) en tres periodos de su registro desde el Pleistoceno hasta la actualidad. Además de una retracción de la extensión del área, las poblaciones muy abundantes de este roedor han pasado a ser extremadamente escasas en la actualidad.

Fig. 6.- Modifications of the distribution area of the arvicolid rodent Microtus (Iberomys) in three recorded periods, from Pleistocene to recent. In addition to a retraction of the area size, the very abundant populations of this rodent in the past become extremely rare in recent times. poblaciones riparias de nuestra península donde se alimentan de juncos en lugares húmedos y boscosos (Ayarzaguena et al., 1976). Se origina tras la diferenciación del primer Microtus eurasiático M. (Allophaiomys) pliocaenicus (Kormos 1933), que invade repentinamente toda la región Holártica a principios del Cuaternario (Cabrera et al., 1983). La historia de Iberomys implica a tres especies sucesivas de un linaje anagenético (ver Ayarzaguena \& López-Martínez, 1976; Cuenca \& Laplana, 1995). La especie más antigua de este linaje, M.(I). huescarensis Mazo et al. 1985, se registra en Granada y Burgos en el Pleistoceno inferior (hace alrededor de $1 \mathrm{Ma}$ ); la especie de edad intermedia M.(I). brecciensis (Giebel 1847) aparece en muchas localidades del centro, este y sur de la Península Ibérica, sur de Francia y noroeste de Italia durante el Pleistoceno medio, y la especie actual M.(I). cabrerae Thomas 1906, se reconoce desde el Pleistoceno superior en la Península Ibérica y sur de Francia. En la Figura 6 se representa en tres mapas sucesivos, desde el Pleistoceno hasta la actualidad, la extensión del área de distribución de Microtus (Iberomys), que muestra una reducción progresiva. El área que ocupaban las abundantes poblaciones de M.(I.) brecciensis durante el Pleistoceno Medio era más extensa y alcanzaba al Norte de los Pirineos centrales y al Este de los Alpes. En el Holoceno antiguo, el área de M.(I.) cabrerae se reduce a la región ibero-occitana (región de clima mediterráneo de la Península Ibérica y Francia). Actualmente está confinado al centro y sur de la Península Ibérica y muestra una importante fragmentación y una reducción progresiva de sus poblaciones, que anuncian su inminente extinción. En la Fig. 7 se recogen las condiciones ambientales de diversas localidades del litoral Mediterráneo distribuidas según dos ejes de índices de pluviosidad y temperatura. En el diagrama se agrupan en un círculo las localidades en que se desarrolla actualmente, o hasta muy recientemente M. cabrerae (Cabrera Millet et al., 1983). Fuera del círculo se indican las localidades donde está hoy extinguido pero donde se desarrollaron sus poblaciones durante el Neolítico, que incluyen lugares como Villena o Almería actualmente en condiciones climáticas de gran aridez muy distintas de las que este roedor requiere: corrientes permanentes y humedales rodeados de bosques. La gran abundancia de $M$. (Iberomys) hace sólo 6.000 años durante el Holoceno antiguo en la cultura neolítica de Los Millares (región de Almería), al igual que la de otros mamíferos 


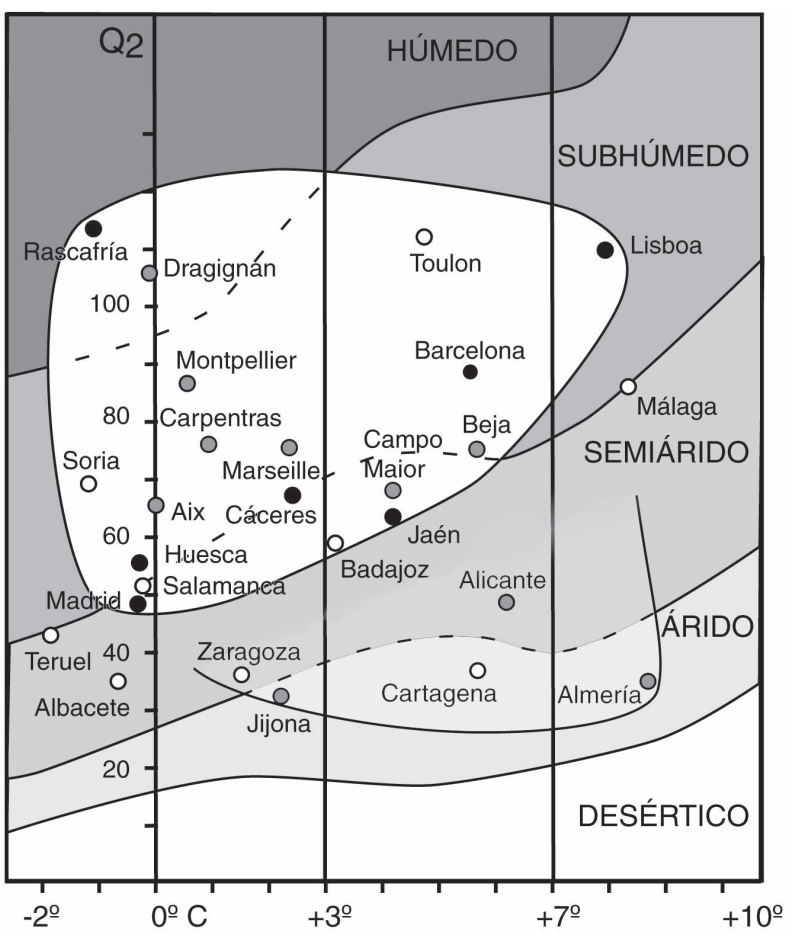

- M. (Iberomys) actualmente presente

- M. (Iberomys) presente hace 6.0000 años

○ M. (Iberomys) actualmente ausente
Fig. 7.- Variaciones de la distribución del roedor Microtus (Iberomys) durante el Cuaternario en varias localidades mediterráneas situadas en un diagrama 'ombroclimático' de Emberger. En círculos negros y área acotada, poblaciones de Iberomys actualmente vivientes. En círculos blancos, localidades donde no se registra. En círculos grises, localidades donde habitaba hace unos 6.000 años (Neolítico). La distribución actual de este roedor en regiones mediterráneas húmedas y frescas permite detectar que las localidades del SE de la Península Ibérica donde habitaba hace 6.000 años han sufrido desde entonces un importante cambio climático, mostrado por su actual posición en condiciones más áridas y cálidas de las que Iberomys puede soportar. Según Cabrera et al. (1983).

Fig. 7.- Variations in the distribution of the rodent Microtus (Iberomys) during the Quaternary in several Mediterranean localities placed in an Emberger's 'ombroclimatic' diagram. In black circles and marked area, recent living populations of Iberomys. In white circles, localities with no records. In grey circles, localities where it dwelled some 6.000 years ago (Neolithic). The recent distribution of this rodent in wet and fresh Mediterranean regions allows to detect an important climatic change in the localities from the SE of the Iberian Peninsula since 6.000 years ago, as shown by their recent position in more arid and warmer conditions than Iberomys can support. After Cabrera et al. (1983). como équidos, ciervos, gamos y jabalíes, indica la intensidad y rapidez de la alteración climática que ha sufrido recientemente la región mediterránea (Figura 7).

Por tanto, la historia paleobiogeográfica de Iberomys muestra una pauta similar a la de Prolagus en la extensión de su área de distribución, de tipo asimétrico (Figura 2B) con una extensión grande de su área al principio y una gradual reducción y fragmentación del área hacia el final que se predice ya muy próximo.

Los dos taxones ibéricos cuyas historias paleobiogeográficas se han resumido tienen diferente diversidad y duración, pertenecen a grupos de mamíferos distintos y han vivido en climas y hábitats diferentes, pero su dinámica biogeográfica muestra pautas similares: la extensión de su área es mayor al principio de su historia, de modo que sus centros de origen no pueden ser señalados con precisión, mientras que la fase de reducción del área, acompañada de su fragmentación, está claramente registrada y su lugar de extinción queda bien localizado. Estos ejemplos tienen semejanza con la dinámica biogeográfica de otras muchas especies del registro fósil (como los foraminíferos planctónicos) y con el "ciclo del taxón" en cuanto a la asimetría del desarrollo y declive del área de distribución.

En ambos casos se puede especular y suponer que la fase de origen local y expansión gradual del linaje no ha quedado adecuadamente registrada, de modo que si se desea preservar la aplicabilidad del modelo simétrico se puede acudir a la conocida hipótesis "ad hoc" de la imperfección del registro fósil. Sin embargo, el registro documenta una pauta asimétrica del principio y del final del taxón representado por datos comparables y sin sesgos particulares. Las observaciones de éstos y otros casos indican que si existe una supuesta fase de aparición local y expansión gradual de un taxón, ésta no se observa claramente en el registro, mientras que sí lo está la fase de contracción y de extinción. No hay razones para suponer que en cada caso el registro fósil de la especie durante la fase de reducción del área sea más completo que el de la fase de expansión, pues la imperfección del registro afectaría por igual a ambas fases. En caso de existir una fase de origen local y expansión gradual, debe ser varios órdenes de magnitud más rápida que la fase de contracción y extinción. Si se acumulan los ejemplos de dinámicas paleobiogeográficas que confirmen esta pauta asimétrica, tal como vimos en los casos de las especies-guías, se entenderán las objeciones planteadas por los autores sobre la escasa operatividad del concepto de centro de origen. 


\section{Conclusión}

El concepto de centro de origen de un taxón puede ser estudiado dentro de un modelo biogeográfico preciso de biogeografía dispersalista, el "modelo simétrico" que predice la aparición local de la nueva especie y su expansión progresiva. Estas dos predicciones se pueden poner a prueba por medio de observaciones sobre especies actuales y sobre fósiles. En especies actuales, la aparición local de una especie no ha sido aún observada, pero el estudio de especies invasoras particulares confirma la capacidad intrínseca de algunas especies de expandir su área según un modelo lineal, a velocidad aproximadamente constante e independiente de factores externos. Los ejemplos muestran que los taxones dispersalistas proceden de un área amplia que ha estado abierta a numerosas interacciones y su centro de origen no puede ser localizado. Para inferirlo se utilizan principalmente tres criterios (1: el área de máxima diversidad, 2: el área de mayor antigüedad paleontológica, y 3) el área de la población con rasgos más primitivos), que se basan en postulados inciertos $\mathrm{y}$ no convergen en una misma dirección.

El modelo simétrico aplicado a la dinámica biogeográfica de la historia de un taxón supone que se producen fases aproximadamente simétricas en el tiempo (Figura 2A): un origen local y una expansión lineal al principio, y una contracción gradual y una extinción local al final. Diversas fuentes de datos llevan a refutar este modelo simétrico, y a proponer en su lugar un modelo asimétrico (Figura 2B), en donde el taxón aparece de forma súbita en una amplia área, mientras que su extinción está precedida de una lenta fase de contracción del área frecuentemente acompañada de su fragmentación.

Los datos que llevan a esta conclusión proceden de:

1) la pauta general no expansiva de las especies endémicas, que no tienden a ampliar su área de origen local y que no muestran capacidad dispersiva. La aparición local de las especies, base del concepto de centro de origen, sólo se ha podido asegurar en los casos de neoendemismos insulares, pero éstos no tienden a la expansión que predice el modelo simétrico.

2) el "ciclo del taxón" observado en especies insulares, en las que el área de la especie colonizadora siempre tiende a reducirse a medida que se produce la diferenciación de especies nuevas, siguiendo un ritmo que muestra regulación intrínseca del proceso.
3) la asimetría de las pautas de aparición (muy rápida, cuasi-sincrónica a escala regional) y de extinción (lenta y diacrónica en distintas poblaciones) en las especies-guías de interés bioestratigráfico del registro fósil.

4) la asimetría en las historias paleobiogeográficas de los taxones, que muestran un área de distribución muy amplia ya desde el principio de su registro, y una larga historia de contracción y fragmentación antes de su extinción.

Por tanto, los datos tienden a refutar la aplicabilidad del modelo simétrico, que implica un centro de origen o aparición local para todos los taxones y la generalización de la dispersión como dinámica biogeográfica común a todas especies. Según las observaciones, los casos de especies invasoras que muestran capacidad intrínseca de expandir su área linealmente, no proceden de un área local sino de una amplia región. Inversamente, los casos de especies neoendémicas que proceden de un centro de origen local, no muestran capacidad de expansión y surgen tras la contracción del área mucho más amplia de sus predecesores eurícoras. Las observaciones apuntan por tanto a un modelo alternativo, con una pauta de asimetría temporal inherente a la dinámica biogeográfica de los taxones: $1^{\circ}$ ) gran extensión regional inicial y $2^{\circ}$ ) subsiguiente tendencia general a la reducción del área de distribución.

\section{AGRADECIMIENTOS}

El Dr. Martín-Piera dedicó su vida a algunos de los temas biogeográficos históricos que tanto nos interesan a los científicos naturalistas de distinta formación, y deseamos reconocer su trabajo desde estas páginas. Agradezco al Dr. Jorge Lobo su paciencia, dedicación y su excelente labor editorial. Al Dr. F. B. Sanchiz y a otro revisor anónimo agradezco sus constructivos comentarios y valiosas sugerencias sobre la primera versión de este artículo. Mi agradecimiento se dirige asimismo a los alumnos que con sus trabajos y reflexiones durante veinte años de cursos de Doctorado de Biogeografía y Paleobiogeografía de las Universidades Autónoma y Complutense de Madrid han contribuido a desarrollar esta apasionante área de estudio. Este trabajo se ha realizado en el marco del proyecto BTE2002-1430 del Ministerio de Ciencia y Tecnología.

\section{Referencias}

Alcover, J. A., SAns, A. \& Palmer, M., 1998. The extent of extinctions of mammals on islands. Journal of Biogeography, 25: 913-918.

Andow, D. A., Kareiva, P. M., Levin, S. A. \& Okubo, A., 1990. Spread of invading organisms. Landscape Ecology, 4: 177-188. 
Avise, J. C., 2000. Phylogeography: the history and formation of species. Harvard University Press. Cambridge \& London. 447 pp.

Ayarzaguena, J., Ibañez, I. \& SAn Miguel, A., 1976. Notas sobre la distribución y ecología de Microtus cabrerae. Doñana, Acta Vertebrata, 3: 109-112.

Ayarzaguena, J. \& LóPez-Martínez, N., 1976. Estudio filogenético y comparativo de Microtus cabrerae y Microtus brecciensis. Doñana, Acta vertebrata, 3: 181-204.

BREMER, K., 1992. Ancestral areas: a cladistic reinterpretation of the center of origin concept. Systematic Biology, 41: 436-445.

Brown, C. W., 1998. http://www.soc.soton.ac.uk/ SOES/STAFF/tt/eh/satbloompics.html

Brown, C. W. \& Yoder, J. A., 1994. Coccolithophorid blooms in the global ocean. Journal of Geophysical Research, 99: 7467-7482.

Brunet, M., Beauvilain, A., Coppens, Y., Heintz, E., Moutaye, A. H. E. \& Pildeam, D., 1995. The first Australopithecine 2.500 kilometers west of the Rift Valley (Chad). Nature, 378: 273-275.

Cabrera Millet, M., LóPez-Martínez, N. \& MichauX, J., 1983. Un exemple de lignée endémique ibéroccitane, les campagnols Microtus brecciensis-M. cabrerae: étude phylogénetique et contexte écologique d'un phénomène évolutif récent. In: E. Buffetaut, J. M. Mazin \& E. Salmon, (eds.). Actes Symposium de Paléontologie Goerge Cuvier, Montbéliard: 69-83.

CARlton, J. T., 1996. Biological invasions and cryptogenic species. Ecology, 77: 1653-1655.

Cheylan, G., 1991. Patterns of Pleistocene turnover, current distribution and speciation among Mediterranean mammals. In: R. H. Grooves \& F. Di Castri (eds.), Biogeography of Mediterranean invasions. Cambridge University Press. Cambridge: 227-262.

Crisci, J. V., Katinas, L. \& Posadas, P., 2000. Introducción a la teoría y práctica de la biogeografía histórica. Sociedad Argentina de Botanica. Buenos Aires. 169 pp.

Croizat, L., 1960. Principia Botanica. Edición del autor. Caracas.

Croizat, L., Nelson, G. \& Rosen, D. E., 1974. Centers of origin and related concepts. Systematic Zoology, 23: $265-287$.

Cuenca, G. \& Laplana, C., 1995. Evolución de Iberomys (Arvicolidae, Rodentia, Mammalia) durante el Cuaternario español. In: G. López, A. Obrador \& E. Vicens (eds.). XI Jornadas de Paleontología, Tremp (Lleida), U.A.B.: 69-74.

Cuvier, G., 1798. Tableau élementaire de l'Histoire Naturelle des animaux. Baudouin. Paris. 710 pp.

D'Antonio, C. M. \& KARK, S., 2002. Impacts and extent of biotic invasions in terrestrial ecosystems. Trends in Ecology and Evolution, 17(5): 202-204.
Dotdrio, I., Perdices, A. \& Machordom, A., 1996. Allozymic variation of the endangered killifish Aphanius iberus and its application to conservation. Environmental Biology of Fishes, 45: 259-271.

EBACH, M. C., 1999. Paralogy and the centre of origin concept. Cladistics, 15: 387-391.

ENGHOFF, H., 1995. Historical biogeography of the Holartic: area relationships, ancestral areas and dispersal of non-marine animals. Cladistics, 11: 223-263.

García-París, M., Good, D. A., Parra-Olea, G. \& Wake, D. B., 2000. Biodiversity of Costa Rican salamanders: Implications of high levels of genetic differentiation and phylogeographic structure for species formation. Proceedings of the National Academy of Sciences of the United States of America, 97(4): 1640-1647.

GINGERICH, P. D., 2000. Paleocene/Eocene boundary and continental vertebrate faunas of Europe and North America. GFF, 122: 57-59.

HAUSDORF, B., 1998. Weighted ancestral area analysis and a solution of the redundant distribution problem. Systematic Biology, 47: 445-456.

HengeVeld, R., 1989. Dynamics of Biological Invasions. Chapman and Hall. London. 160 pp.

Hengeveld, R., 1990. Dynamic Biogeography. Cambridge University Press. Cambridge. 249 pp.

Howard, D. J. \& Berlocher, S. H. (eds.), 1998. Endless Forms: Species and Speciation. Oxford University Press. New York. 500 pp.

Humphries, C. J., 2002. Review of Bruce S. Lieberman's "Paleobiogeography: Using Fossils to Study Global Change, Plate Tectonics, and Evolution" Journal of Paleontology; 76: 1110-1112.

JENKINS, D. G., 1992. Predicting extinctions of some extant planktic foraminifera. Marine Micropaleontology, 19: 239-243.

JenKINS, D. G., 1994. Predicting extinctions. In: E. Molina (ed.). Extinción y registro fósil. S.I. Universidad de Zaragoza. Cuadernos Interdisciplinares, 5: 219-227.

Kooljman, S. A. L. M., 1993. Dynamic energy budgets in biological systems. Cambridge University Press. Cambridge. 350 pp.

Krause, D. W. \& MAAS, M. C., 1990. The biogeographic origins of late Paleocene-early Eocene mammalian immigrants of the Western Interior of North America. Geological Society of America, Special Papers, 243: 71-105.

LANG, M., 1990. Phylogenetic analysis of the genus group Tracheloptychus-Zonosaurus (Reptilia, Gerrhosauridae) with a hypothesis of biogeographical unit relationships in Madagascar. In: G. Peters \& R. Hutterer (Eds.): Vertebrates in the tropics. Museum Alexander Koenig. Bonn: 261-274.

Lewis, M. A. \& Pacala, S., 2000. Modeling and analysis of stochastic invasion processes. Journal of Mathematical Biology, 41: 387-429. 
LÓPEZ-MARTíNEZ, N., 2001. Paleobiogeographical history of Prolagus, an European ochotonid (Lagomorpha). Lynx (Praha), n.s., 32: 215-231.

López-Martínez, N. \& Truyols, J., 1994. Paleontología: conceptos y métodos. Síntesis. Madrid. 334 pp.

Martín Piera, F. \& SANMARtín, I., 1999. Biogeografía de áreas y biogeografía de artrópodos holárticos y mediterráneos. Boletín de la Sociedad Entomológica Aragonesa, 26: 535-560.

McDonald, J. F. \& Ayala, F. J., 1974. Genetic response to environmental heterogeneity. Nature, 250: 572-574.

Mooney, H. A. \& Cleland, E. E., 2001. The evolutionary impact of invasive species. Proceedings of the National Academy of Sciences of the United States of America, 98: 5446-5451.

Morrone, J. J. \& CRISCI, J. V., 1995. Historical biogeography: Introduction to methods. Annual Review of Ecology and Systematics, 26: 373-401.

Moyá-Solá, S., Quintana, J., Alcover, J. A. \& KöHLER, M., 1999. Endemic island faunas of the Mediterranean Miocene. In: G. Rössner \& K. Heissig (eds.). The Miocene land mammals of Europe. Munchner Geowissenschaftliche Abhandlungen, 435-442.

Nelson, G. \& Platnick, N. I., 1981. Systematics and Biogeography: Cladistics and Vicariance. Columbia University Press. New York. 567 pp.

Nelson, G., \& Rosen, D. E. (eds.), 1981. Vicariance Biogeography: A Critique. Columbia University Press. New York. 593 pp.

Oosterbroek, P. \& Arntzen, J. W., 1992. Area-cladograms of circum-mediterranean taxa in relation to Mediterranean paleogeography. Journal of Biogeography, 19: 3-20.

Otte, D. \& Endler, J. A. (eds.), 1989. Speciation and it's consequenses. Academy of Natural Sciences. Philadelphia. 679 pp.

PAUL, C. R. C., 1987. Stratigraphic mensuration. Lethaia, 20: 256.

Platnick, N. L, \& Nelson, G., 1978. A method of analysis for historical biogeography. Systematic Zoology, 27: $1-16$.

Por, F. D., 1978. Lessepsian migration: The influx of Red Sea biota into the Mediterranean by way of the Suez Canal. Ecological studies, 23: 1-228.

Repetur, C. P., Van Welzen, P. C., \& De Vogel, E. F., 1997. Phylogeny and historical biogeography of the genus Bromheadia (Orchidaceae). Systematic Botany, 22: 465-477.
Ricklefs, R. E. \& Bermingham, E., 2002. The concept of taxon cycle in biogeography. Global Ecology and Biogeography, 11: 353-361.

RickleFs, R. E. \& Cox, G. W., 1972. Taxon cycles in the West Indian avifauna. American Naturalist, 106: 195-219.

RonQuist, F., 1994. Ancestral areas and parsimony. Systematic Biology, 43: 267-274.

SÁIz, F., 1973. Biogeography of soil beetles in Mediterranean regions. In: F. di Castri \& H.A. Mooney (eds.). Mediterranean type ecosystems. Origin and structure. Springer Verlag. New York: 285-294.

SANCHIZ, F. B., 1979. Aportes al método de delimitación de regiones en biogeografía histórica. I Reunión Nacional de Biogeografia, Guadalajara, 1977: 224-243.

SPOOR, C. F. \& SondAAR, P. Y., 1986. Human fossils from the endemic island fauna of Sardinia. Journal of Human Evolution, 15: 399-408.

Uerpmann, H.-P., 1987. The Origins and Relations of Neolithic Sheep and Goats in the Western Mediterranean. In: J. Guilaine, J. Courtin, J. Roudil \& J. L. Vernet (eds.), Premières communautés paysannes en Méditerranée Occidentale. CNRS. Paris: 175-179.

VAn den Bosch, F., Metz, J. A. J. \& Diekmann, O., 1990. The velocity of spatial population expansion. Journal of Mathematical Biology, 28: 529-565.

VAN DER MADE, J., 1999. Intercontinental relationship Europe-Africa and the Indian Subcontinent. In: G. Rössner \& K. Heissig (eds.). The Miocene Land Mammals of Europe. Verlag Dr. Friedrich Pfeil. Munchen: 457-472.

VAN VALEN, L., 1965. Morphological variation and width of ecological niche. American Naturalist, 99: 377390 .

VoelKer, G., 1999. Dispersal, vicariance, and clocks: Historical Biogeography and speciation in a cosmopolitan Passerine genus. Evolution, 53: 1536-1552.

Westbroek, P., 1991. Life as a geological force. Norton. New York. 240 pp.

Wiley, E. O., 1988. Vicariance Biogeography. Annual Review of Ecology and Systematics, 19: 513-542.

WiLson, E. O., 1961. The nature of the taxon cycle in the Melanesian ant fauna. American Naturalist, 95: 169-193. 